Supporting information for

\title{
Mesoporous Silica as Sorbents and Enzymatic Nanoreactors for Microbial Membrane Proteomics
}

Jinzhi Zhao ${ }^{1}$, Ruijun Jian ${ }^{1}$, Yuning Wang ${ }^{1}$, Beibei Yang ${ }^{1,2}$, Dan Zhao ${ }^{3}$, Chengpin Shen ${ }^{3}$, Liang Qiao ${ }^{1, *}$ and Baohong Liu ${ }^{1, *}$

1. Department of Chemistry, and Shanghai Stomatological Hospital, Fudan University, Shanghai 200000, China.

2. Department of Chemistry and Chemical Engineering, Nantong University, Nantong 226000, China

3. Shanghai Omicsolution Co., Ltd., Shanghai 201100, China

\section{Table of Contents}

Supplementary experimental section

S-2

The identified OVA peptides with different proteolysis methods (Table S1)

The mixing ratio of mixed bacteria and the number of identified proteins (Table S2)

The ratio of identified membrane proteins to all membrane proteins of the species in the mixed bacteria sample by the Met-SBA-15 (Table S3)

Adsorption of BSA and OVA by SBA-15 and Met-SBA-15 (Fig. S1)

Comparison of OVA digestion using different proteolysis methods (Fig. S2)

Number of proteins identified from E. coli and mixed bacterial (Fig. S3)

The distribution of the GRAVY of the identified non-membrane proteins (Fig. S4) 


\section{Supplementary experimental section}

LC-MS/MS analysis. The digested peptides of bacterial membrane proteins were dissolved in solvent A ( $0.1 \%$ formic acid in water) and analyzed by on-line nanospray LC-MS/MS on an Orbitrap Fusion Lumos Tribrid mass spectrometer (Thermo Fisher Scientific, Waltham, MA, USA) coupled to an EASY-nanoLC 1200 system (Thermo Fisher Scientific). Three $\mu \mathrm{L}(1 \mu \mathrm{g})$ peptides was loaded to an analytical column of Acclaim PepMap $\mathrm{C}_{18}(75 \mu \mathrm{m} \times 25 \mathrm{~cm})$ and separated with a 60 min gradient for $E$. coli (0-50 min, 5 to $45 \%$ of buffer B $(0.1 \%$ formic acid in $\mathrm{ACN}) ; 50-55 \mathrm{~min}, 45 \%$ to $80 \%$ buffer B; $55-60 \mathrm{~min}, 80 \%$ of buffer B) or a 130 min gradient for the mixed bacterial sample (0-5 min 4\% of buffer B; 5-120 min, 4 to $50 \%$ of buffer B; $120-121 \mathrm{~min}, 50 \%$ to $95 \%$ buffer $\mathrm{B} ; 121-130 \mathrm{~min}, 95 \%$ of buffer $\mathrm{B}$ ). The column flow rate was maintained at $250 \mathrm{~nL} / \mathrm{min}$ with the column temperature of $40^{\circ} \mathrm{C}$. The electrospray voltage of $2 \mathrm{kV}$ versus the inlet of the mass spectrometer was used. The mass spectrometer was operated in a data dependent acquisition (DDA) mode to switch automatically between MS and MS/MS. Parameters were as follows: (1) MS: scan range $(\mathrm{m} / \mathrm{z})=350-1200$; resolution $=120,000 ;$ AGC target $=8 \mathrm{e} 5 ;$ maximum injection time $=50 \mathrm{~ms}$; include charge states $=2-7$; exclusion duration $=30 \mathrm{~s}$; (2) $\mathrm{HCD}-\mathrm{MS} / \mathrm{MS}$ : resolution $=15,000$; isolation window $=4 ;$ AGC target $=50,000 ;$ maximum injection time $=25 \mathrm{~ms} ;$ collision energy $=30$.

Database search. The LC-MS/MS data were processed by PEAKS Studio (version X+, Bioinformatics Solutions Inc., Waterloo, Canada). PEAKS DB was set up to search against the UniProt-Proteomes Escherichia coli (ver. 202006, 5062 entries), Bacteroides fragilis strain ATCC 25285 (ver. 202006, 4234 entries), Enterobacter asburiae (ver. 202006, 5254 entries), Klebsiella aerogenes strain ATCC 13048 (ver. 
202006, 4909 entries), Klebsiella pneumoniae (ver. 202006, 5126 entries), Morganella morganii (ver. 202006, 3510 entries), Pseudomonas aeruginosa (ver. 202006, 5564 entries) and Citrobacter freundii (ver. 202006, 5149 entries), with trypsin as enzyme, and maximum missed cleavages as 2 . The fragment ion mass tolerance was set as 0.02 $\mathrm{Da}$ and the parental ion tolerance was set as $7 \mathrm{ppm}$. Carbamidomethylation (C) was specified as a fixed modification. Oxidation (M), deamidation (NQ) and acetylation (K) were specified as variable modifications. The false discovery rate (FDR) cut-off on both peptide and protein level was $1 \%$ by using a target-decoy strategy. Proteins were identified based on at least one unique peptide.

Bioinformatic analysis. Transmembrane domains (TMDs) of identified proteins were predicted by the TMHMM algorithm (http://www.cbs.dtu.dk/services/TMHMM/) ${ }^{12}$, in which proteins with at least one TMD was regarded as integrated membrane proteins (transmembrane protein). The grand average of hydrophobicity (GRAVY) values of proteins were calculated by the ProtParam program (https://web.expasy.org/protparam/) ${ }^{12}$. 
Table S1. The identified OVA (Uniprot ID: P01012) peptides with different proteolysis methods.

\begin{tabular}{|c|c|c|c|c|c|c|c|}
\hline \multirow{2}{*}{ Sequence $^{[\mathrm{a}]}$} & \multirow{2}{*}{$m / z$} & \multicolumn{3}{|c|}{ Met-SBA-15 } & \multicolumn{3}{|c|}{ In-solution } \\
\hline & & $20 \mathrm{~min}$ & $2 \mathrm{~h}$ & $12 \mathrm{~h}$ & $20 \mathrm{~min}$ & $2 \mathrm{~h}$ & $12 \mathrm{~h}$ \\
\hline OVA183-187 & 632 & $\sqrt{ }$ & $\sqrt{ }$ & $\sqrt{ }$ & & & $\sqrt{ }$ \\
\hline OVA281-285 & 647 & $\sqrt{ }$ & $\sqrt{ }$ & $\sqrt{ }$ & & & \\
\hline OVA106-111 & 780 & $\sqrt{ }$ & $\sqrt{ }$ & $\sqrt{ }$ & & & $\sqrt{ }$ \\
\hline OVA279-285 & 889 & & & $\sqrt{ }$ & & & $\sqrt{ }$ \\
\hline OVA48-56 & 1063 & $\sqrt{ }$ & $\sqrt{ }$ & $\sqrt{ }$ & & & \\
\hline OVA191-200 & 1210 & & $\sqrt{ }$ & $\sqrt{ }$ & & & \\
\hline OVA52-62 & 1348 & $\sqrt{ }$ & $\sqrt{ }$ & $\sqrt{ }$ & $\sqrt{ }$ & $\sqrt{ }$ & $\sqrt{ }$ \\
\hline OVA48-59 & 1417 & $\sqrt{ }$ & $\sqrt{ }$ & $\sqrt{ }$ & $\sqrt{ }$ & $\sqrt{ }$ & $\sqrt{ }$ \\
\hline OVA188-200 & 1556 & $\sqrt{ }$ & $\sqrt{ }$ & $\sqrt{ }$ & $\sqrt{ }$ & $\sqrt{ }$ & $\sqrt{ }$ \\
\hline OVA265-277 & 1582 & $\sqrt{ }$ & $\sqrt{ }$ & $\sqrt{ }$ & $\sqrt{ }$ & $\sqrt{ }$ & $\sqrt{ }$ \\
\hline OVA128-143 & 1688 & $\sqrt{ }$ & $\sqrt{ }$ & $\sqrt{ }$ & $\sqrt{ }$ & $\sqrt{ }$ & $\sqrt{ }$ \\
\hline OVA265-278 & 1710 & $\sqrt{ }$ & $\sqrt{ }$ & $\sqrt{ }$ & & & $\sqrt{ }$ \\
\hline OVA324-340 & 1774 & $\sqrt{ }$ & $\sqrt{ }$ & $\sqrt{ }$ & & & $\sqrt{ }$ \\
\hline OVA1-17 & 1840 & $\sqrt{ }$ & $\sqrt{ }$ & $\sqrt{ }$ & & & \\
\hline OVA144-159 & 1859 & $\sqrt{ }$ & $\sqrt{ }$ & $\sqrt{ }$ & $\sqrt{ }$ & $\sqrt{ }$ & $\sqrt{ }$ \\
\hline OVA265-280 & 1951 & & & $\sqrt{ }$ & & & \\
\hline OVA341-360 & 2009 & $\sqrt{ }$ & $\sqrt{ }$ & $\sqrt{ }$ & & & \\
\hline OVA 106-123 & 2227 & & & $\sqrt{ }$ & $\sqrt{ }$ & $\sqrt{ }$ & \\
\hline OVA124-143 & 2249 & & $\sqrt{ }$ & $\sqrt{ }$ & $\sqrt{ }$ & $\sqrt{ }$ & \\
\hline OVA201-219 & 2284 & $\sqrt{ }$ & $\sqrt{ }$ & $\sqrt{ }$ & $\sqrt{ }$ & $\sqrt{ }$ & $\sqrt{ }$ \\
\hline OVA160-182 & 2460 & $\sqrt{ }$ & & & $\sqrt{ }$ & $\sqrt{ }$ & \\
\hline
\end{tabular}

[a] The position of the amino acid in the ovalbumin (OVA) protein sequence. 
Table S2. The amount of eight strains in the mixture, and the number of identified membrane proteins and all proteins of each strain using different proteolysis methods.

\begin{tabular}{|c|c|c|c|c|c|c|c|}
\hline \multirow{2}{*}{ Bacteria } & \multirow{2}{*}{$\begin{array}{l}\times 10^{8} \\
\mathrm{CFU}\end{array}$} & \multicolumn{3}{|c|}{ Met-SBA-15 } & \multicolumn{3}{|c|}{ In-solution } \\
\hline & & $\begin{array}{c}\text { membrane } \\
\text { proteins } \\
\left(\mathrm{N}_{\mathrm{m}}\right)\end{array}$ & $\begin{array}{c}\text { all } \\
\text { proteins } \\
\left(\mathrm{N}_{\mathrm{a}}\right)\end{array}$ & $\mathrm{N}_{\mathrm{m}} / \mathrm{N}_{\mathrm{a}}$ & $\begin{array}{c}\text { membrane } \\
\text { proteins } \\
\left(\mathrm{N}_{\mathrm{m}}\right)\end{array}$ & $\begin{array}{l}\text { all } \\
\text { proteins } \\
\left(\mathrm{N}_{\mathrm{m}}\right)\end{array}$ & $\mathrm{N}_{\mathrm{m}} / \mathrm{N}_{\mathrm{a}}$ \\
\hline E. coli & 3.33 & 175 & 799 & $21.9 \%$ & 65 & 766 & $8.5 \%$ \\
\hline C. freundii & 10 & 194 & 929 & $20.9 \%$ & 66 & 739 & $8.9 \%$ \\
\hline B. fragilis & 10 & 164 & 855 & $19.2 \%$ & 44 & 348 & $12.6 \%$ \\
\hline E. asburiae & 10 & 137 & 656 & $20.9 \%$ & 51 & 555 & $9.2 \%$ \\
\hline K. aerogene & 10 & 156 & 756 & $20.6 \%$ & 57 & 564 & $10.1 \%$ \\
\hline K. pneumonia & 20 & 136 & 703 & $19.3 \%$ & 52 & 743 & $7.0 \%$ \\
\hline M. morganii & 20 & 197 & 996 & $19.8 \%$ & 67 & 880 & $7.6 \%$ \\
\hline P. aeruginosa & 20 & 236 & 1213 & $19.5 \%$ & 75 & 947 & $7.9 \%$ \\
\hline
\end{tabular}


Table S3. The ratio of identified membrane proteins to all possible membrane proteins of the species in the mixed bacteria sample using the Met-SBA-15 proteolysis method.

\begin{tabular}{cccc}
\hline Bacteria & membrane proteins $\left(\mathrm{N}_{\mathrm{m}}\right)$ & $\begin{array}{c}\text { all membrane } \\
\text { proteins }\left(\mathrm{N}_{\mathrm{a}}\right)\end{array}$ & $\mathrm{N}_{\mathrm{m}} / \mathrm{N}_{\mathrm{a}}$ \\
\hline E. coli & 175 & 1118 & $15.65 \%$ \\
C. freundii & 194 & 1251 & $15.51 \%$ \\
B. fragilis & 164 & 1065 & $15.40 \%$ \\
E. asburiae & 137 & 1217 & $11.26 \%$ \\
K. aerogene & 156 & 1221 & $12.78 \%$ \\
K. pneumonia & 136 & 1245 & $10.92 \%$ \\
M. morganii & 197 & 874 & $22.54 \%$ \\
P. aeruginosa & 236 & 1342 & $17.59 \%$ \\
\hline
\end{tabular}



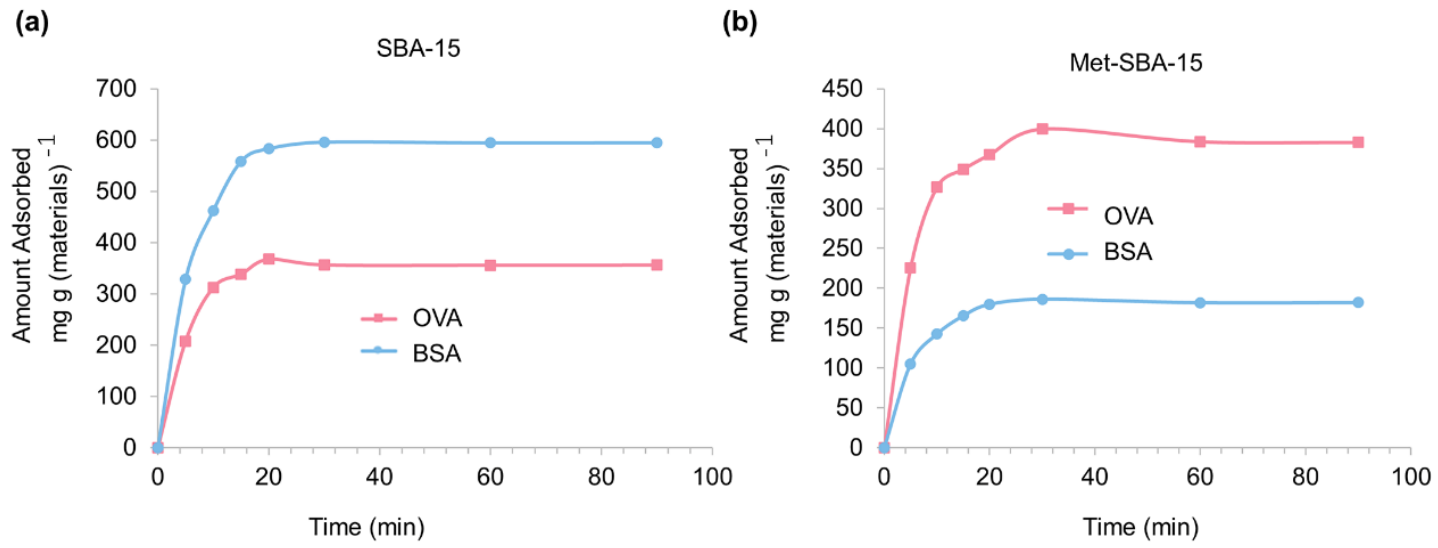

Figure S1. Adsorption of BSA (1 mg/mL) and OVA (1 mg/mL) by the (a) SBA-15 (1 $\mathrm{mg} / \mathrm{mL})$, (b) Met-SBA-15 (1 mg/mL) as function of incubation time. 
(a)

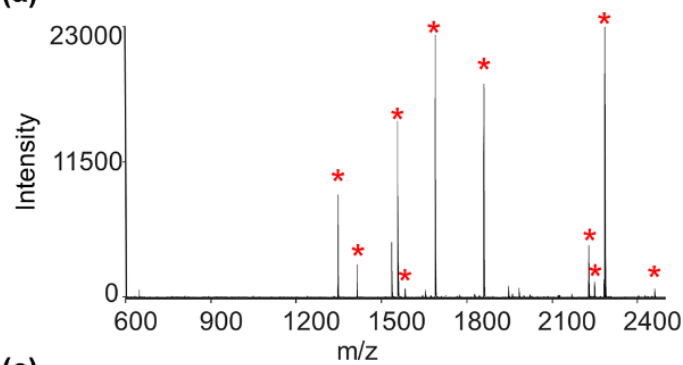

(c)

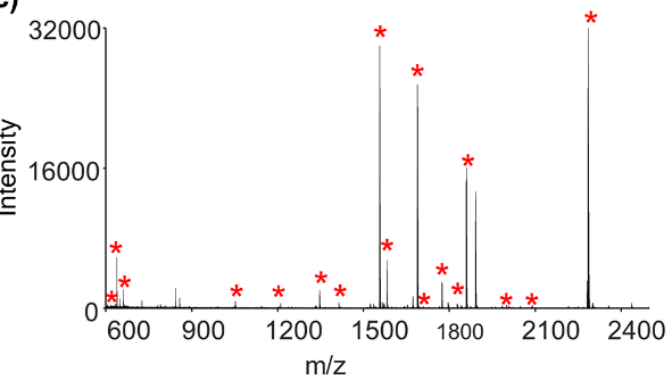

(e)

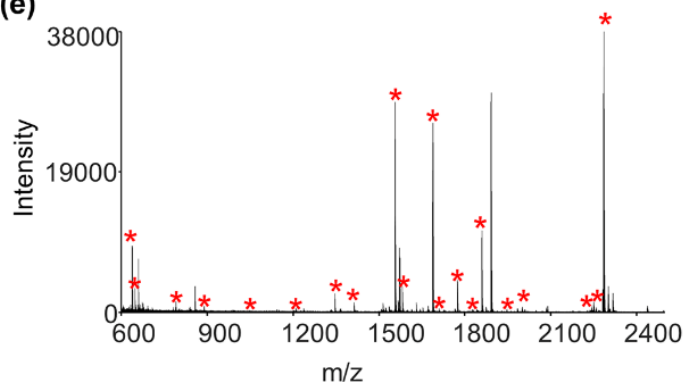

(b)

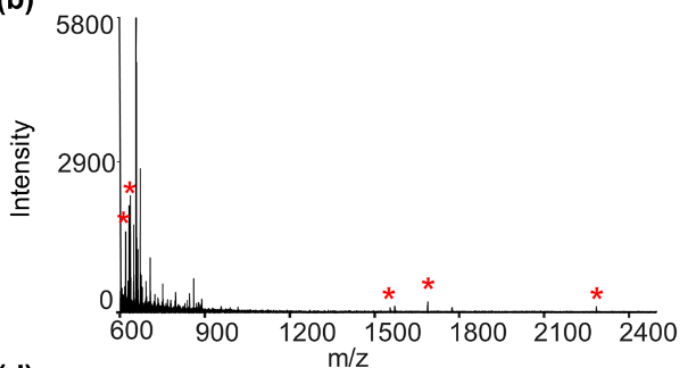

(d)
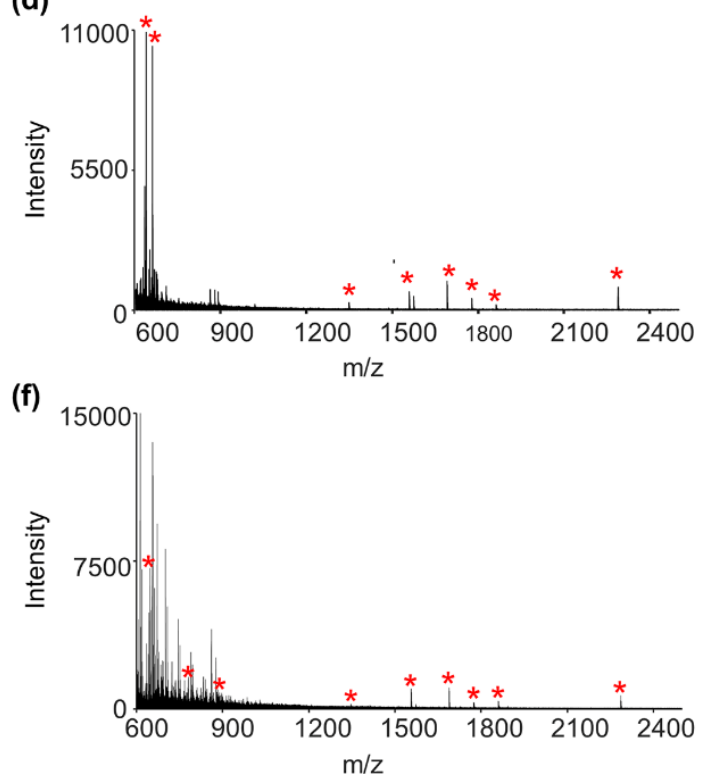

Figure S2. Comparison of OVA digestion using the in-solution and the Met-SBA-15 assisted digestion protocols. Peptide mass fingerprinting spectra of OVA $(0.1 \mathrm{mg} / \mathrm{mL})$ after (a) 2 hours of in-solution digestion, (b) 20 min of Met-SBA-15 assisted digestion (elute solution), (c) 2 hours of Met-SBA-15 assisted digestion (supernatant), (d) 2 hours of Met-SBA-15 assisted digestion (elute solution), (e) 12 hours of Met-SBA-15 assisted digestion (supernatant), and (f) 12 hours of Met-SBA-15 assisted digestion (elute solution). 
(a)

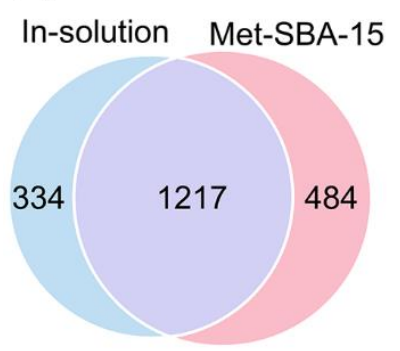

E. coli (b)

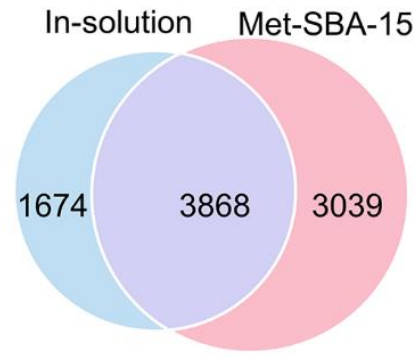

mixed bacteria

Figure S3. Number of protein groups identified from (a) E. coli and (b) mixed bacterial sample of 8 strains using the Met-SBA-15 assisted proteolysis and in-solution digestion. 
(a)

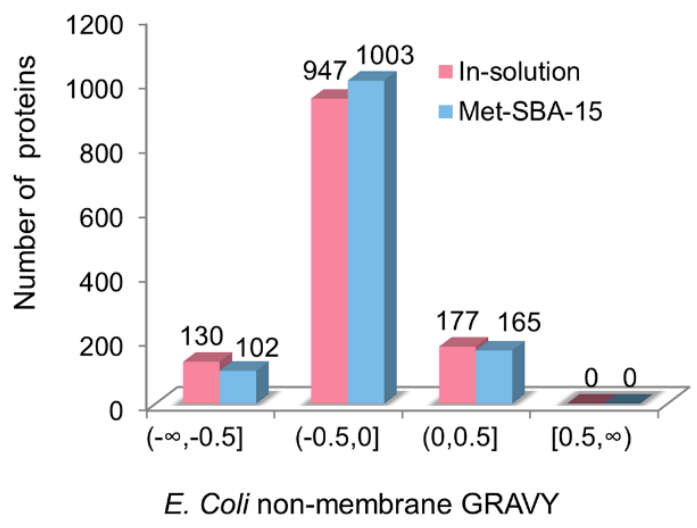

(b)

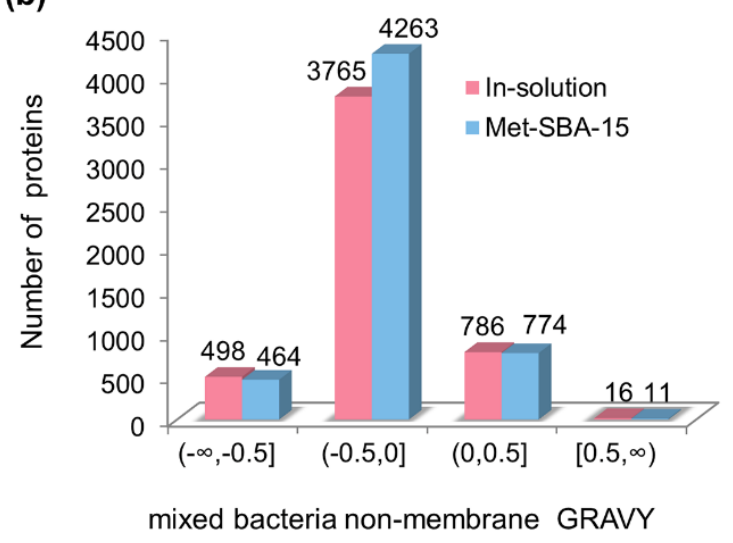

Figure S4. The distribution of the Grand Average of Hydrophobicity (GRAVY) of the identified non-membrane proteins from (a) E. coli and (b) mixed bacterial sample using the in-solution proteolysis or the Met-SBA-15 assisted proteolysis. 